\title{
Designing Digital Comprehensive System to TEST AND ASSESS THE INTELLIGENTLY BEHAVIORS OF FROM 6 TO 12 YEARS OLD CHILDREN BASED ON THE WECHSLER INTELLIGENCE THEORY
}

\author{
Yaser Rahmani ${ }^{1}$ and Ahmad Habibizad Navin ${ }^{2}$ \\ ${ }^{1}$ Department of Computer, Science and Research Branch, Islamic Azad University, East \\ Azerbaijan, Iran \\ ${ }^{2}$ Department of Computer, Tabriz Branch, Islamic Azad University, Tabriz, Iran
}

\begin{abstract}
Intelligence Quotient (IQ) is a ratio achieved by dividing the mental age over chronological age. There are several ways to achieve a mental age and usually professionals use certain tests. In order to avoid having decimals and facilitate calculations, the result of mental age/chronological age ration is multiplied by 100. $I Q$ tests are used to determine IQ value. Tests and methods available to determine IQ have shortcomings such as time-consuming nature of the tests, non-comprehensive tests, non-systematic tests, etc. This article seeks to design a comprehensive digital system for testing and evaluating children's IQ according to Wechsler's Intelligence Theory to solve problems of the tests and methods available.
\end{abstract}

\section{KEYWORDS}

Comprehensive Digital System, IQ, Intelligence Test, Wechsler's Intelligence Theory.

\section{INTRODUCTION}

Checking the past shows that men have been always trying to know others so that they can correspond to their actions with them, but it was not scientific. Over time and with the development of human, family, and community needs, methods to measure abilities such as intellectual abilities and behaviours are spread faster and gain scientific aspect [1]. The first effective steps to make measurement tools occur in the laboratory of Wilhelm Maximilian Wundt. It can be said that the efforts to correctly measure phenomena are the establishment of appropriate techniques for the analysis of reactions, preparation of norms, and use of mathematics to interpret the results; the necessary steps to prepare the tests were for the first time in the laboratory of Wundt [2].

After Wundt, Sir Francis Galton took effective measures. He in his famous book called Hereditary Genius, tried to calculate genius by calculation of the deviation each person can have from the average of the group. Galton noted the differences' distribution around the mean and took effective steps in order to prepare questionnaires to assess personality [3].

In 1890, Mckeen Cattell, an American psychologist, for the first time raised the psychological testing that has been translated into Persian as the psychological test. Mckeen Cattell prepared 10 tests and gave them to their students and determined individual differences based on the 
responses. The psychologist's tests had two major disadvantages: they measured low level abilities, and the other had no norms [4]. The actual preparation of tests started in 1905 by Alfred Binet and Theodor Simon. They provided a number of questions with increasing difficulty degree and gave them to different age groups, to estimate children's ability. The questions that were known as scale of intelligence measurement were revised again in the years 1908 and 1911. The last revision gave way to most countries. In America, Professor Terman, at the University of Stanford, standardized Binet test with the necessary changes for the American community and named it Stanford-Binet test [1]. In section 2 of this paper, we study test methods and measuring of children intelligence. In section 3, we explain the design of new methods and in section 4, we implement new methods. By using the proposed system, we measured number of children in Baneh City that in section 5, we analyzed the results. Finally, in section 6, we discuss about the conclusion and also suggestion for further study.

\section{THE STUDY OF TEST AND ASSESSMENT METHODS OF CHILDREN'S INTELLIGENCE}

There are different theories and methods to test and measure the intelligence of children including Kohs cubes test, Thorndike intelligence test, Porteus labyrinthine routes, Goodenough dummy test, Wechsler's Intelligence Scale, Raven intelligence test, etc; a certain number of these methods are discussed here.

\subsection{Kohs Cubes Test}

Kohs cubes test measures practical intelligence in children 5 to 15 years old. Cubes test was produced in 1920 by Mr. Kohs; it is a solution to reduce the failure in verbal tests, when used alone. This test is of practical tests made to avoid interference of language in the measurement of intelligence. In this test, different shapes with different colors are printed on cards; the test taker must make the shapes using cubes. To make each shape, a definite time period is set to make the desired shape using cubes. Based on the time period of creating the shapes, the scores will be different. In the end, all scores obtained from each of the shapes are added and IQ is achieved with the use of chronological age and mental age [5].

\subsection{Dummy Good enough Test}

Folrence Good enough developed this test, in which the kid is asked to draw a picture of a man. In the Good enough dummy test, time is not considered; using a pencil, a kid only draws a picture of a person on paper. After completing the drawing, in scoring, only the present components are considered, not the beauty of the drawing; the flexibility is considered as well. In this test, it is important that the components are fully and clearly drawn. For example, if the finger is not included in drawing the hand, the test is scored lower. And if the fingers are completely drawn, more scores are given to the test taker. The obtained score is converted to an IQ value via a table, mental age, and Ms. Stern's method. The test is common and does not depend on cultural factors. Good enough's test has been adapted to many languages and most cultures. Drawing is a good way to study children's intelligence, and usually good students are among good drawing students; however, the drawing test cannot be accounted as a full IQ scale. This test is a tool that is added to the traditional tests [6].

\subsection{Wechsler Intelligence Scale}

David Wechsler in the 1930s began studying a number of standard tests. To make his initial scale set, he selected 11 subtests. A number of his subtests were taken from different parts of the 1937Stanford-Binet revised test. The rest of the subtests were obtained from the group exams of the 
army, design of Kohs's cubes, alpha military test, beta military test, test of completion of Haley Pictures, and Pinter-Patterson test. These subtests were combined and published in 1939 as Wechsler-Bellevue Intelligence Scale [7]. The initial scale of Wechsler-Bellevue was built for adults including two parts: verbal scale and performance measure; in addition to separate scores, they had a score of Intelligence Quotient. But, in 1949, Wechsler formulated Wechsler's Intelligence Scale for children so that with this test, the intelligence of children of older than 5 years were measured the same way as adults [7].

\subsection{Emotional Intelligence Questionnaire (Managerial)}

The test was introduced in 1996 by Golman. This test indicates the intelligence rate to sense the emotions of others and the ability or to continue friendship relationships with others, accredited by the British Psychological Society. It has 69 steps; usually between 15 to 30 minutes is intended to do the test. The test is verbal, and during the test stages, the questions are asked as in a questionnaire. Based on the responses, the intelligence rate is measured at the end. Emotional intelligence test contains seven separate parts [8]. The seven elements are briefly described in this section.

$>$ Awareness: the ability to understand and manage and control our own feelings, and to believe, manage, and control oneself in a working environment.

$>$ Flexibility: ability to hang on and adapt oneself to the conditions and pressures.

$>$ Individual intelligence: the ability to be aware of the needs and expectations of others to solve the problems.

$>$ Influence: the ability to persuade others by knowing and understanding the needs.

$>$ Certainty intuition: the ability to reach a clear conclusion on the basis of vague and incomplete information.

$>$ Conscience: the ability to encourage others to deal with problems and choose the correct path.

$>$ Motives: the desire and passion to get the right results and the impact upon it.

\subsection{Trait of Emotional Intelligence Questionnaire}

The test was published in2013 by Dr. Petrides, child psychologist and member of teaching psychology association. It is a method for multi-purpose measurement and evaluation of emotional intelligence. It is a verbal test and, during the test, questions are asked as in a questionnaire; at the end, intelligence is measured based on the responses. It includes 153 steps based on seven planned stages. The test can be performed on paper or online, as online, is more practical. This application contains all of the life and work management, talent, leadership trait, measurement of the power to choose, team creation, and so on. It is accredited by the British Psychological Society [9]. The whole score is a function of general intelligence based on four main factors that will be discussed in this section briefly.

$>$ Welfare: The amount of happiness and the amount of which a person is positive.

$>$ Self-control: how much a person can take control his stress?

$>$ Excitement: The capacity to understand, express emotions, maintain, and keep a friendship relationship with others in the community.

$>$ Social: social skills of a person, that is, how could they communicate with others?

\section{Designing A NeW Method}

A new method is designed in this section. First, new ideas, innovations and new work incentives are mentioned. Then, on the basis of them, a new approach is designed formally and described via a graph. In the design of the proposed system, there are three tests parameters of mental tester and 
test takers' administrator. Each of these factors in the system carries out special operations. We want to measure and assess the amount of intelligent behaviors of children, 6 to 12 years old, in suggested system. For this purpose, we should define standard tests in the terms of validity and reliability in the system. Then, the child log in to the system and do the defined tests. The results of tests based on mental tester viewpoint and also the saved data are analyzed in the data base; the amount of intelligence and intelligent behaviors is achieved. Result tests are saved in database in section of result test that is related to every child who log in to the system and take the test. After taking the test, child and parents can log in to the system and observe the results. In Figure 1, Use Case Diagram is shown that the test taker performs it in the system. The test taker shall have the possibility to register in the system, to $\log$ in and start doing the test; the results of previous tests must be provided for the test taker.

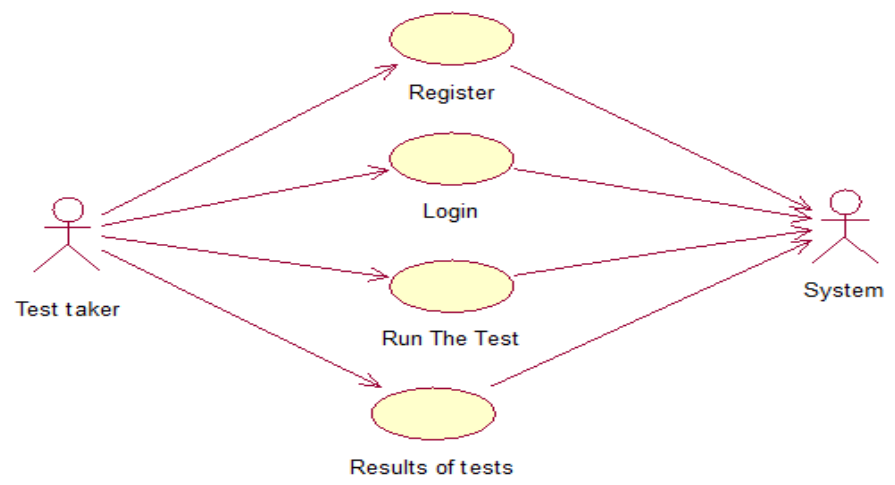

Figure 1. Use Case Diagram of the test taker in the proposed system

In Figure 2, Use Case Diagram of mental detector is shown in the system. The mental tester should be able to log in and edit specifications of the test and the test taker information. Mental tester, by selecting the test, should inform the test taker which test to perform; in some cases, the mental tester must be involved in test scoring; to see the results of tests carried out; provide reports of tests and test takers and send the results of the test takers to the administrator.

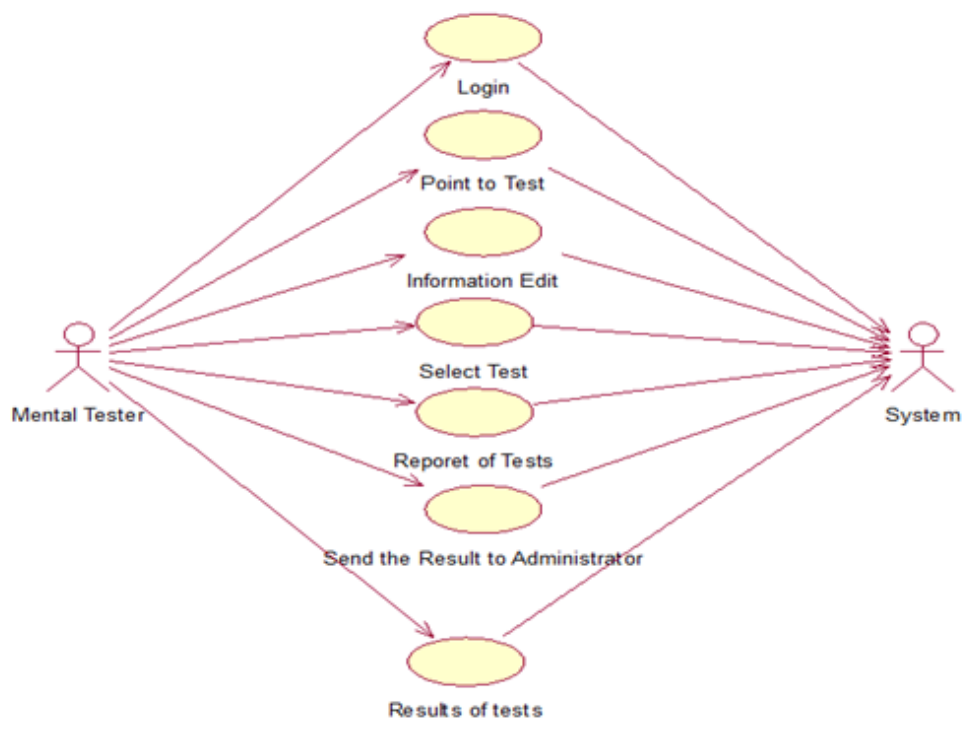

Figure 2. Use Case Diagram of mental tester in the proposed system 
Figure 3 shows the Use Case Diagram of the test taker of administrator in the proposed system. Only the administrator can $\log$ in the system and view the results of his test taker.

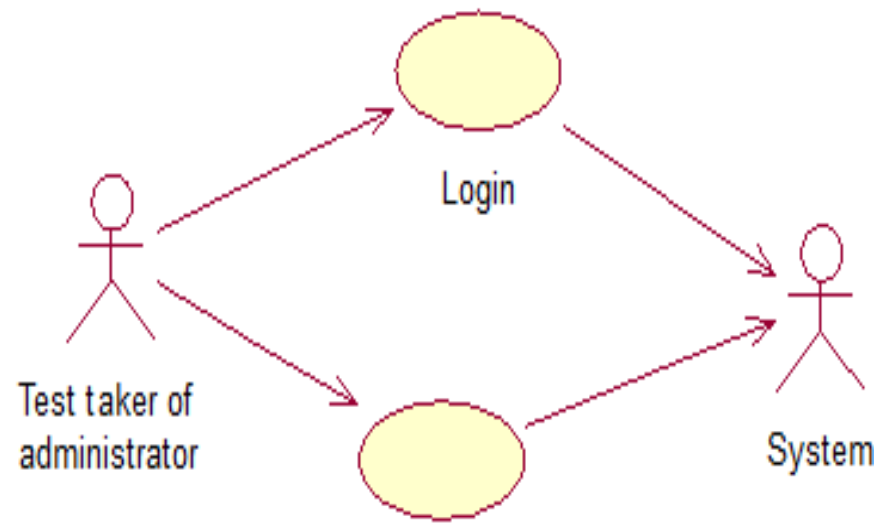

Results of tests

Figure 3.Use Case Diagram of the test takers of administrator in the proposed system

Apart from the Use Case, the mental tester and the test taker interact in the proposed system, and the test taker uses the mental tester guidelines. In Figure 4, Use Case Diagram of mental tester and the test taker are displayed.

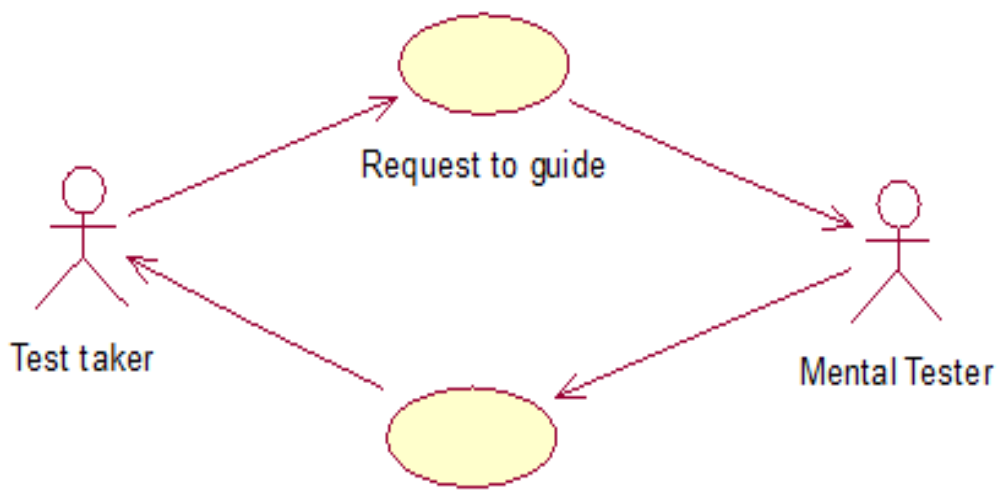

Replay Requset

Figure 4. Use Case Diagram between mental tester and the test taker in the proposed system

In the proposed system, there is the possibility of registration of the test taker data and the tests, automatically calculating the scores, and display of scores of each test. In Figure 5, Use Case Diagram of the proposed system is shown. 
International Journal in Foundations of Computer Science \& Technology (IJFCST) Vol.6, No.1, January 2016

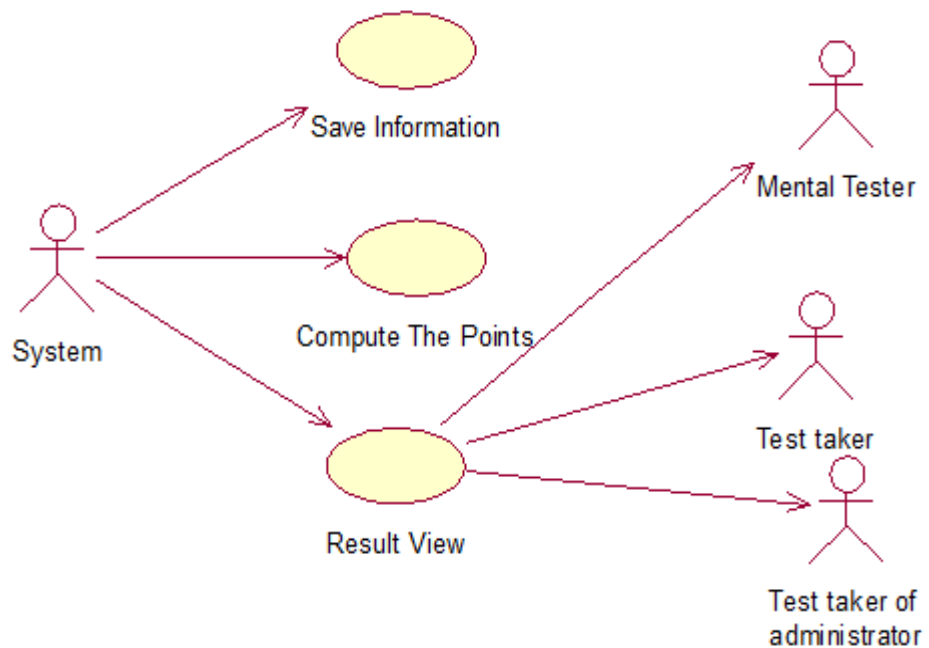

Figure 5. Use Case Diagram of the proposed system

In Figure 6, general Use Case Diagram of the proposed system is displayed.

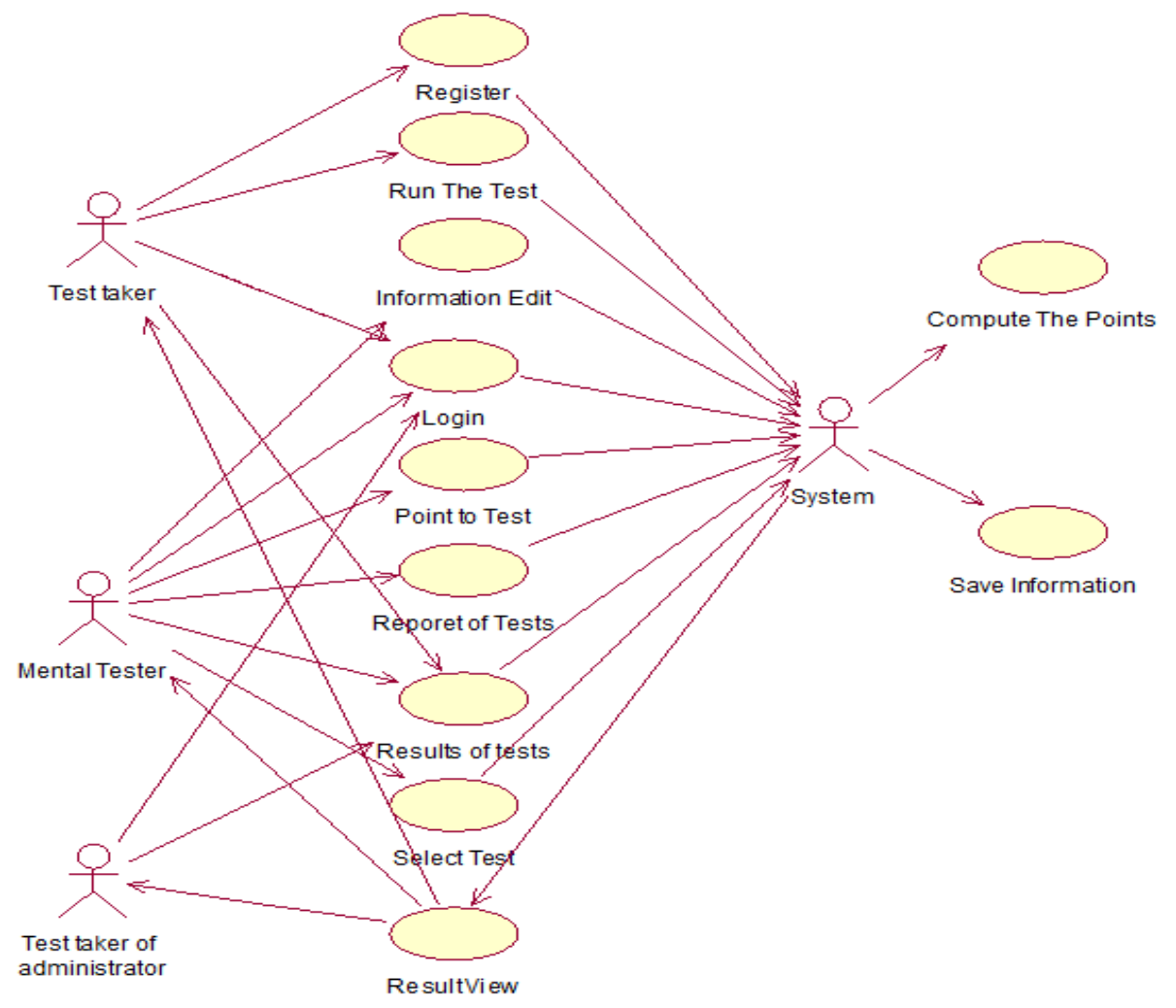

Figure 6. General Use Case Diagram of the proposed system 
In Figure 7, Sequential Diagram of registration processes in the system, system $\log$ in, administration of tests, saving the test results, and sending the results to the test administrator are shown.

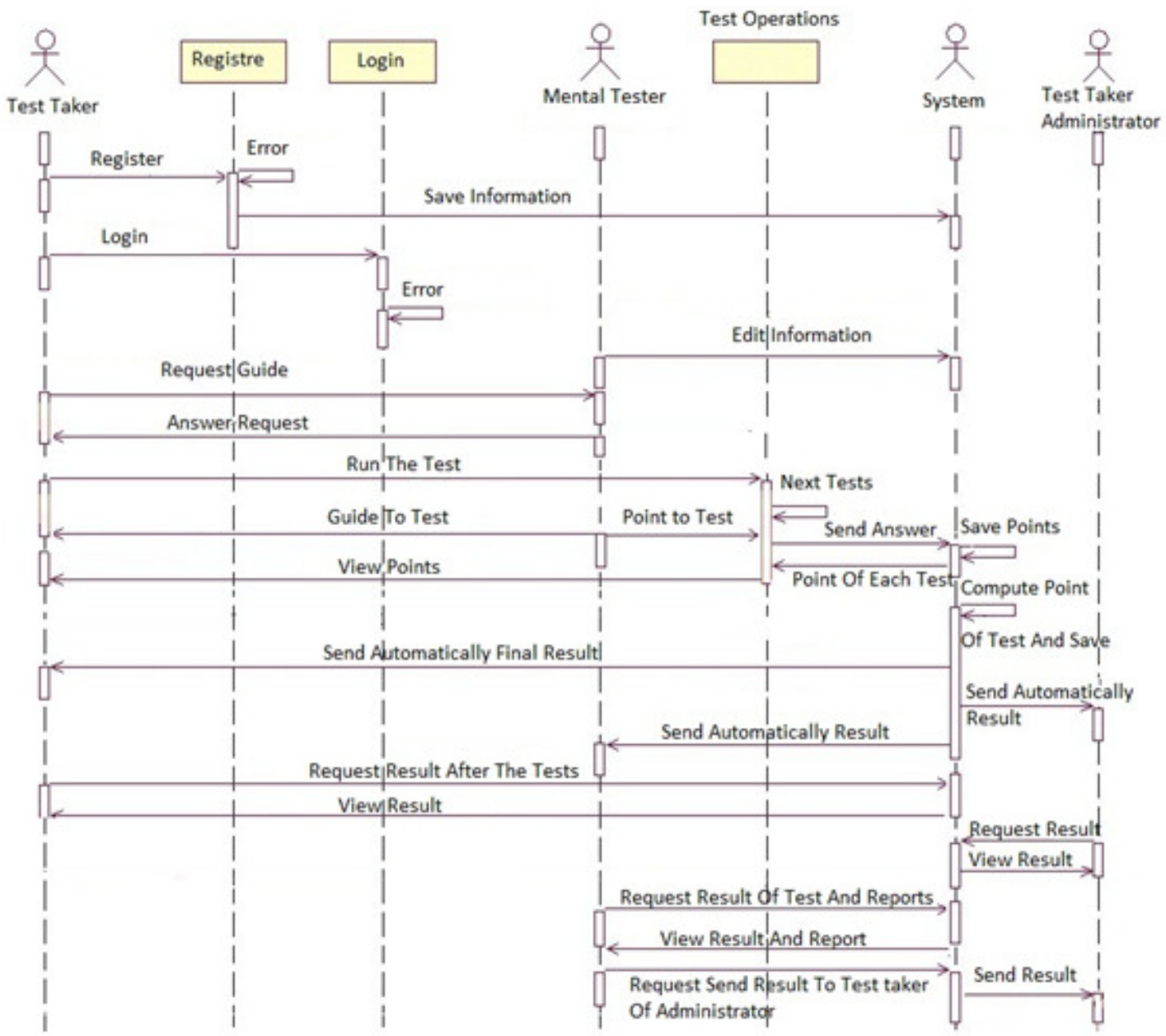

Figure 7. Sequential Diagram of the proposed system

\section{THE IMPLEMENTATION OF THE NEW METHOD}

For implementation of the designed proposed system, ASP.Net environment, C \# programming language, and also the SQL Server database have been used. In Figure 8, the user entry page is shown as an example of the system implementation. Users can select tests and view the scores of each test stage. 


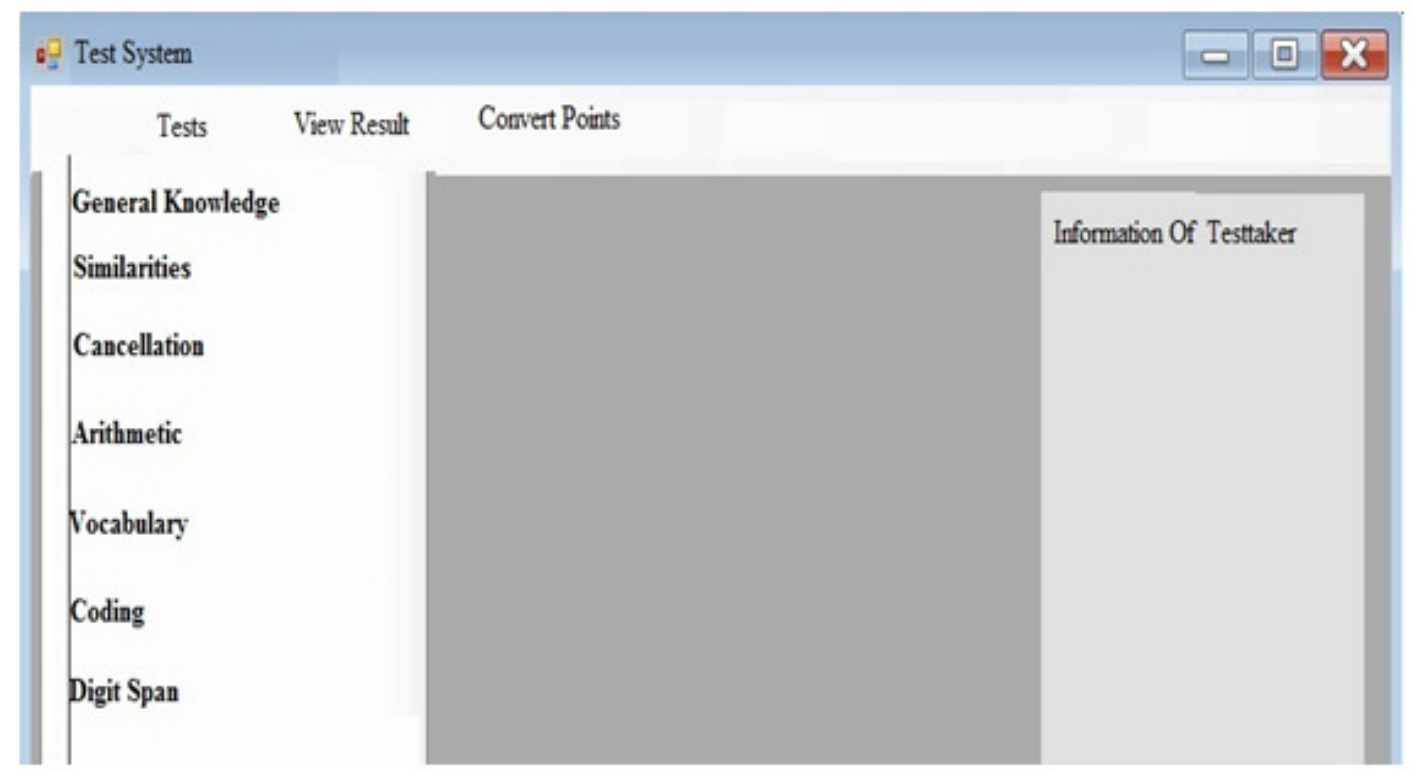

Figure 8 . The test run page of the proposed system

We have used Wechsler intelligence theory in this system. Given that this system is comprehensive, so the tests can be changed, and other intellectual theories can be used. Wechsler's third version for children includes 12 subtests the results of which are ultimately recorded in the system. Using the "tests" menu, the subtests can be administered in order. Figure 9 shows an example of the tortuous routes subtest. At each stage of subtest, specifications of the test taker are also displayed. Some subtests need mental tester guide so that the test taker takes advantage of it at the test administration time. Some subtests are time-dependent, and the test taker must complete them in a certain amount of time, and the sooner the subtest finishes, more points are scored. Wechsler' third version for children measures both verbal intelligence and nonverbal intelligence (practical intelligence) and obtains general intelligence using verbal and nonverbal intelligence.

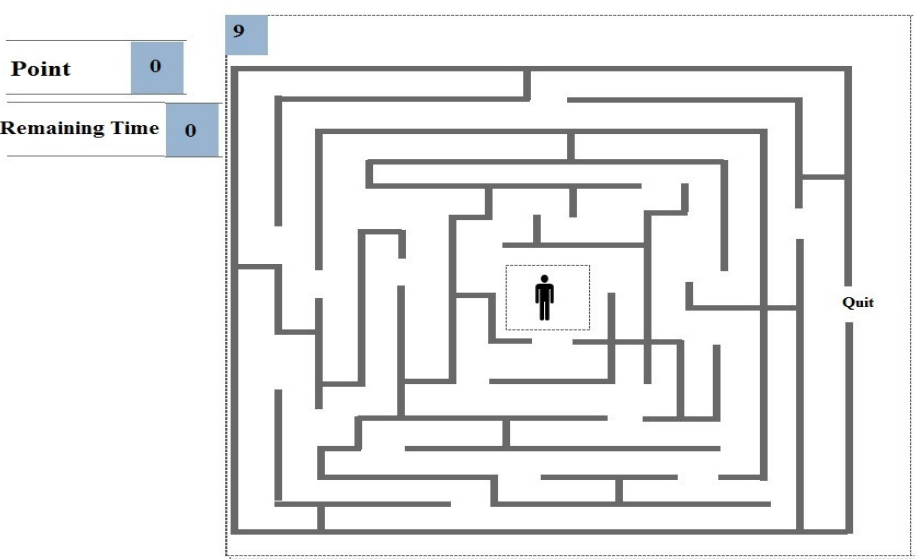

Figure 9. An example of Wechsler subtest (tortuous routes test) 
After administration of the subtests, the results are recorded in the database. The obtained results of subtests are raw results.

Scores in some tests are raw date and must be converted to standardized scores, and then IQ is achieved. Determining IQ is based on pre-determined tables by the theorists. As is evident in Figures 9, there is a menu of "scores' conversion". Clicking on "raw scores' conversion" key, the results of subtests are converted to standardized scores. Ultimately, the system obtains the overall standardized scores and determines the IQ, and each person's IQ is saved along with specifications. The test administration method, scoring each subtest, the conversion of raw scores to standardized scores, and determination of the IQ is done using the Wechsler Intelligence theory.

\section{RESULTS AND DISCUSSION}

According to Figure 10, it is clear that, the tests administration time significantly decreased using the proposed system. This process will increase according to the number of test takers.

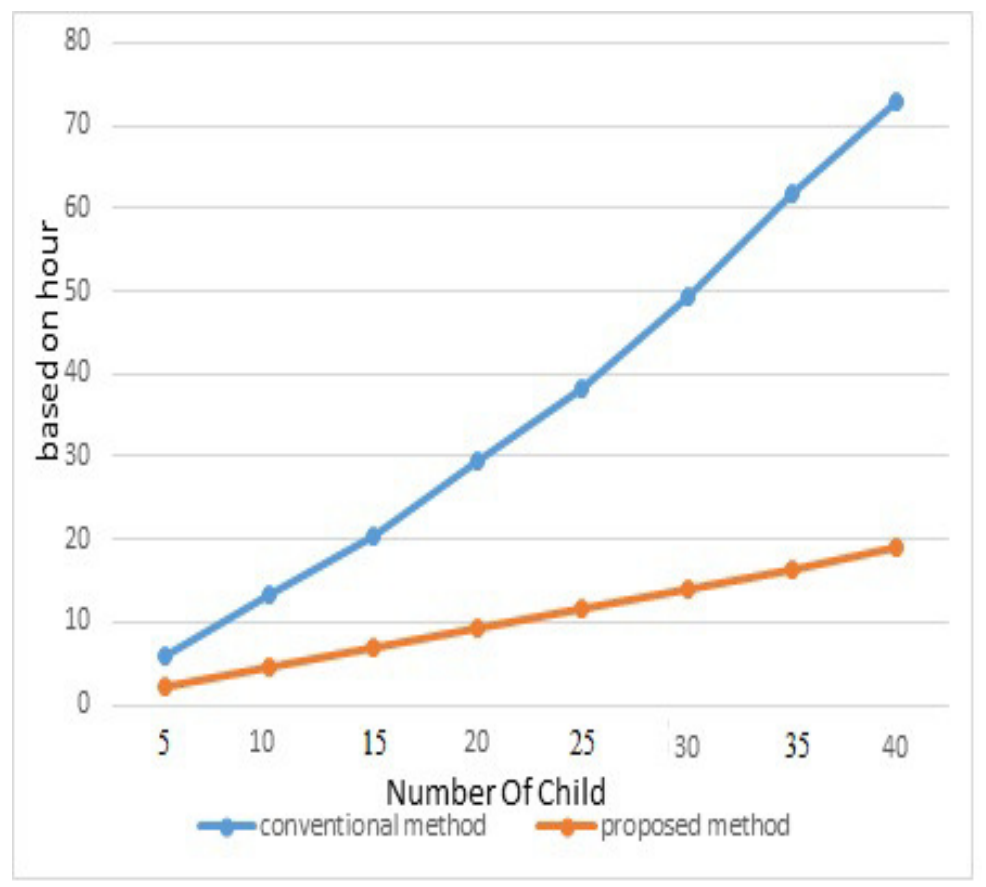

Figure 10. Chart of comparing the test administration time by the conventional method and the proposed method based on hour

Using the designed system, the IQ of 40 primary school students of Baneh city, Iran, was measured.

In 2014 February, the test was performed manually and traditionally. To administer the test and convert raw scores to standard scores and obtaining IQ and preparing and distributing required materials and tools to test 40 students, 73 hours was spent. After 7 months in August 2015, the test was administered on the 40 students, using the designed system. 18.9 hours was spent to administer the test on the 40 students. It is clear that there was a great time reduction. In the traditional method, a place is needed for maintaining the specifications and data related to the students, tests, and results. This need was addressed in the proposed method, and all the 
specifications and data of the students, tests, and results were recorded on storage medium and documented. After administering the traditional tests, searching and finding tests and test results as well as specifications and related data of a student was very difficult, but using the designed system, finding the required documents are easily done, and reporting and publishing them in the proposed system is easy. As shown in Figure 11, the time required to search for and report information, using the proposed system, is much less than the traditional method.

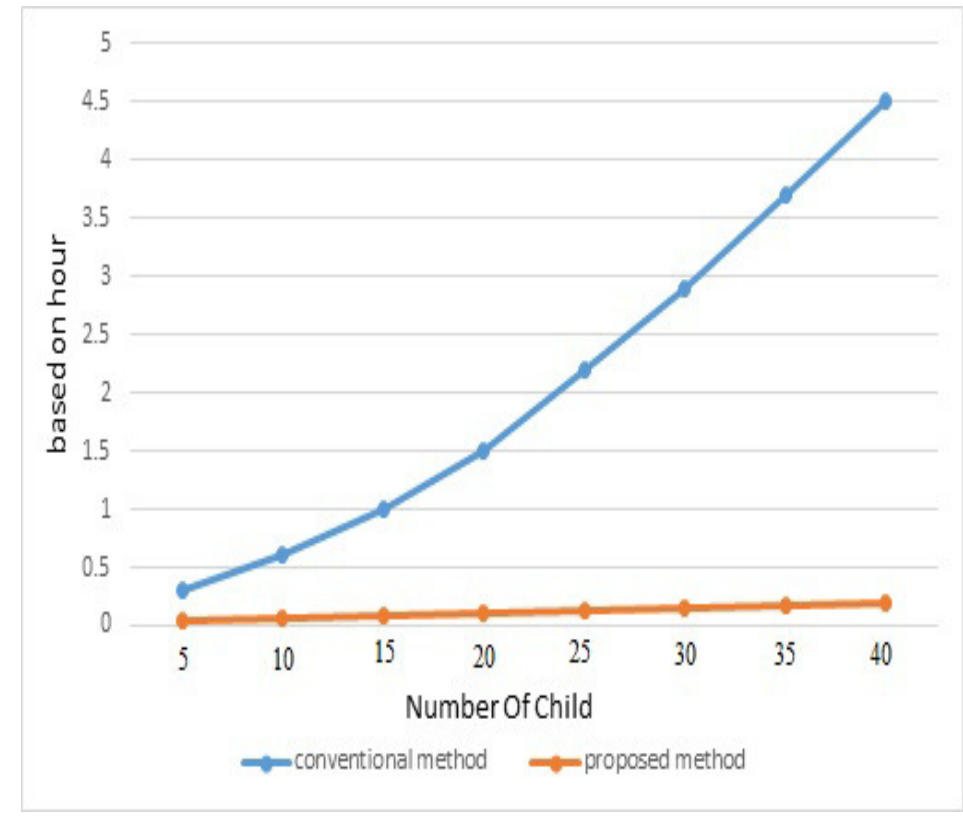

Figure 11. Chart of comparing the search time and reporting in the traditional method and the proposed method based on hour

Also, the edition of profile specifications and information of students was done with less time and cost. Moreover, notification and sending the results to the students' supervisors took place at the time of test administration.

\section{CONCLUSIONS AND FUTURE WORKS}

In this research study, a comprehensive digital system was designed to test and measure intelligent behaviors of children 6 to 12 years old based on the Wechsler's intelligence theory. Common methods for testing and measuring intelligent behaviors and IQ of children have several advantages including greater flexibility in scoring and more interaction of the tester and the test taker. A new system was proposed, designed, and implemented to resolve the disadvantages of high cost and time to administer the test, storage and maintenance of the test results and specifications and information of individuals, searching, reporting, and analysis of results. The results of use of the proposed new system which was conducted on 40 students of primary school in Baneh city showed its improvement. Researches that can be done in the future can be Designing Digital Comprehensive System to measure adult intelligence, Designing Digital Comprehensive System to measure personality and social behaviors of people, improving the designed system by using cloud environment and changing the designed system for using in cell phones and portable devices. 
International Journal in Foundations of Computer Science \& Technology (IJFCST) Vol.6, No.1, January 2016

\section{REFERENCES}

[1] H. Ganji, M. Sabet, "Psychometric (the theoretical foundations of psychological tests)", Savalan, Version 3, 264 pages, 2003.

[2] L. Sprung, "Wundt, Wilhelm Maximilian (1832-1920)", International Encyclopedia of the Social \& Behavioral Sciences, pp.792-795, 2015.

[3] J.C.W. Waller, "Galton, Sir Francis (1822-1911)", International Encyclopedia of the Social \& Behavioral Sciences, doi:10.1016/B0-08-043076-7/00246-1, PP. 5860-5863, 2001.

[4] D. P. Schultz, S. E. Schultz, "A history of modern psychology", version 10, Harcourt Brace Jovanovich, 0155374656, 9780155374652, 403 pages, 1987.

[5] R. Paulette, C. Denis, "Strategy development in a block design task", Vol. 30, No. 1, Intelligence, pp.1-25, 2002.

[6] J.L. Jolly, "Florence L. Goodenough: Portrait of a Psychologist", Roeper Review, Vol.32, No.2, pp.98-105, 2010.

[7] G. Groth-Marnat, "Handbook of Psychological Assessment", version 3, John Wiley \& Sons, 0471052205, 9780471052203, 816 pages, 1997.

[8] V. Dulewicz, M. Higgs,'Emotional Intelligence Questionnaire (Managerial)”, GL Assessment, 2009. URL:http://ptc.bps.org.uk/test-registration-test-reviews/search-test-reviews/viewtestsummary/40

[9] K. V. Petrides, "Trait Emotional Intelligence Questionnaire", Thomas International Ltd, 2013. URL:http://ptc.bps.org.uk/test-registration-test-reviews/search-test-reviews/viewtestsummary/ 270 